\title{
PROKLA-Redaktion
}

\section{Internationale Organisationen 50 Jahre nach Bretton Woods}

Vor genau 50 Jahren wurde in Bretton Woods die ökonomische Grundlage der damals neuen Weltordnung für die Zeit nach der Niederschlagung des Faschismus gelegt. Die frustrierenden Erfahrungen mit dem Völkerbund der Zwischenkriegszeit vor Augen, noch geschockt von den Folgen des Zusammenbruchs des Weltmarkts nach der großen Weltwirtschaftskrise, sollten die Rahmenbedingungen einer zukünftig expandierenden Weltwirtschaft geschaffen werden. Das Vertrauen in die Selbstheilungskräfte des Marktes war in jener Phase nur gering. Regulation ökonomischer Prozesse gegen die zerstörerischen Wirkungen der Marktkräfte, auch auf internationaler Ebene, stand auf der Tagesordnung. Zwar verabschiedeten die Vereinten Nationen auf ihrer Gründungsversammlung in San Francisco kein Dokument über die wirtschaftlichen und sozialen Grundrechte oder gar über eine »gerechte《 Weltwirtschaftsordnung; es war jedoch allen beteiligten Akteuren bewußt, daß die internationale ökonomische Zusammenarbeit auf ein neues und tragfähiges Fundament gestellt werden muBte.

So entstand das internationale Währungssystem stabiler Wechselkurse mit dem Internationalen Währungsfonds, der für den Ausgleich von Leistungsbilanzdefiziten zur Stabilisierung des Kursgefüges sorgen sollte. Eine Bank für Wiederaufbau und Entwickung des zerstörten Europa wurde gegründet, die später als Weltbank in erster Linie die Finanzierung der Entwickung der Länder der Dritten Welt organisieren sollte. Die ebenfalls geplante Welthandelsordnung kam auf der Konferenz von Havanna 1947 dann doch nicht zustande; die USA ratifizierten das Abkommen nicht. Das allgemeine Zoll- und Handelsabkommen jedoch nahm im Verlauf seiner Entwicklung, obwohl dazu gar nicht ausgelegt, den Charakter einer Welthandelsorganisation an, den es auch zum 1. Januar 1995 förmlich erhalten wird: Das GATT wird in die zum Abschluß der »Uruguay-Run- 
de $\ll$ in Marakesch beschlossene Welthandelsorganisation WTO überführt.

Die neuen Prinzipien des Zusammenlebens der Völker nach zwei Weltkriegen hießen: politische Gleichheit und freier Handel. Die Entfachung des Kalten Krieges kurz nach dem Ende des Zweiten Weltkriegs verschob jedoch die Akzente, die in den Debatten um das $\gg$ Grand Design« der Nachkriegsordnung gesetzt worden waren. Die kapitalistische Welt definierte sich als »freier Westen«, die realsozialistischen Länder bildeten das »sozialistische Lager«. Die Grenzlinie der gespaltenen Welt verlief mitten durch Deutschland. Vor 45 Jahren entstanden die Bundesrepublik Deutschland als Teil des »freien Westens « und die Deutsche Demokratische Republik als Teil des »sozialistischen Lagers «.

Die Weltordnung der Nachkriegsjahrzehnte war einerseits ausgesprochen übersichtlich. Der Ost-WestKonflikt erzeugte auf einfache Weise naheliegende Feindbilder. Im Schwarz-Weiß-Schema von Freund und Feind bzw. in der Rot-BiauKennung der NATO-Manöverstrategen) war die Orientierung einfach - geradezu eine Carl Schmittsche Konstellation. Beide Blöcke bildeten ihre eigenen Ordnungen heraus, und sie versuchten, ihre jeweiligen Ordnungsprinzipien in die noch nicht festgelegte, weil zum Teil noch gar nicht dekolonialisierte Welt zu exportieren. »Westerniza- tion« und »Modernisierung « waren die Parolen des »freien Westens «, »Sozialismus « und ebenfalls »Modernisierung « die Versprechen, die aus dem Osten kamen. 50 Jahre später kann man freilich Bilanz ziehen. Die Feststellung ist im Jahre 1994 nicht überraschend, daß der Westen sein Modell erfolgreicher exportierte als der realsozialistische Widerpart, auch wenn zu Recht bezweifelt wird, daß alle Welt das Modell der kapitalistischen Industriegesellschaft umsetzen kann, ohne die natürliche Tragfähigkeit der Erde zu überlasten. Und zudem hat sich der Markt zwar als leistunsgstarker Allokationsmechanismus erwiesen doch ist er nicht in der Lage, Armut und Elend zu beseitigen und für einen sozialen Ausgleich zu sorgen, weder innerhalb nationaler Marktwirtschaften noch in der Weltwirtschaft als Ganzer.

\section{I.}

Die »sanfte Revolution « in Osteuropa, Perestroika und Glasnost in der Sowjetunion und deren schließliche Auflösung in die Gemeinschaft Unabhängiger Staaten im Dezember 1991 beendeten die Übersichtlichkeit des »Systemwettbewerbs «. Nach kurzer Euphorie wird der $\gg$ freie Westen $\ll$ seines $»$ Siegs im Kalten Krieg « am »Ende der Geschichte in der »neuen Weltordnung « nicht so recht froh. Der Globus erscheint dem Beobachter chaotisiert und von Turbulenzen geschüttelt. Vorbei ist das »unipolar 
moment «, von dem Krauthammer während des Truppenaufmarsches am Golf sprach. Die Rede von der neuen Weltordnung, die Präsident Bush im September 1990 zum ersten Mal schwang, klingt wenige Jahre später trotz des militärischen Bombasmus am Golf, trotz des immer noch bedrohlichen Overkills und trotz des finanziellen und ökonomischen Gewichts der USA in der Weltgesellschaft eher kleinlaut, zumindest hohl. Denn offensichtlich kann selbst die Supermacht USA ihre »Leadership« nur noch mit »Partnern« ausüben. Mit dem äußeren Feind scheint paradoxerweise den Siegern die Politik verlustig gegangen zu sein, und dies in einem Moment, in dem durch die Auflösung einer Weltmacht - ganz im Gegensatz zu dem unsinnigen Geschwätz vom »Ende der Geschichte - eine völlig unerwartete Wiederkehr von Geschichte, Politik und Entwicklungsdynamik stattfindet. Also muß Politik neu erfunden werden. Die internationale Ordnung, die sich derzeit herausbildet, erscheint weniger als Resultat bewußter politischer Projektion denn als eine »Fundsache« nicht-intendierten Handelns von Nationalstaaten, internationalen Organisationen aber auch von privatwirtschaftlichen Machtkonglomeraten wie multinationalen Konzernen und transnational operierenden Banken. Sogar die Nicht-Regierungsorganisationen haben zwischenzeitlich die höchste aller Politik-Ebenen, die globale nämlich, erklommen und melden sich dort zu Wort (siehe den Artikel von Karl Bruckmeier).

Die Institutionen, die während des Ost-West-Konfliktes Erwartungssicherheit ermöglichten, passen ihren Inhalt, ihre Funktion und zum Teil auch ihre Struktur an die neuen Lagen seit 1989 an. Die Anpassungsprozesse antworten jedoch nicht auf die Hoffnungen nach 1989, die hinsichtlich der Herausbildung einer je nationalen und möglicherweise auch internationalen Zivilgesellschaft sicher überzogen waren. Ernüchterung hält Einzug. Denn die zu bewältigenden Aufgaben sind gewaltig: Die der UNO bei der Friedensschaffung und Friedenserhaltung zugeschriebene Rolle ist angesichts der neuen Konfliktdimensionen in der tradierten Organisation von Politikprozessen kaum zu erfüllen. Die Bedeutung der souveränen Nationalstaaten hat sich angesichts der Globalisierung von ökonomischen Prozessen und ökologischen Folgewirkungen ökonomischen Handelns so sehr relativiert, daß vielfach vom Ende des Nationalstaats gesprochen wird. Im Gegensatz zu dieser Beobachtung zeigen sich jedoch in besonders deutlichem Maße gerade in Europa neo-nationalistische, regionalistische Tendenzen, die gleichsam als Nachtseite der Globalisierung von Politik, Ökonomie, Kultur das scheinbar freundliche Bild nach der »Abwahl eines Jahrhunderts « (Ash) verdunkeln. 
Auf dem Weltmarkt sind die Gegensätze nicht etwa geringer geworden, so wie es die neoklassische Gleichgewichts- und Modernisierungstheorie versprach, sondern sie sind gewachsen (zum Zivilisationsverlust des modernen Kapitalismus vgl. PROKLA 85, Dezember 1991). Zugleich haben sich die Trennungslinien zwischen armen und reichen, stagnierenden und prosperierenden Ländern und Regionen verschoben. Bis Ende der 80er Jahre waren wir gewohnt, über den Norden, den Süden und den Osten zu sprechen. Diese eindeutigen Grenzziehungen sind in Bewegung geraten. Ein nicht unerheblicher Teil des Ostens, so ist zu befürchten, wird auf das ökonomisch-soziale Niveau des Suidens herabsinken. Der traditionelle Süden hat sich längst beachtlich ausdifferneziert; entsprechend groß sind auch die Interessenunterschiede zwischen Ländern wie Burkina Faso oder Somalia auf der einen, den berühmten asiatischen Tigern auf der anderen Seite. Dieser Prozeß hat große Auswirkungen auf das internationale Kräftegleichgewicht und die Handlungsfähigkeit innerhalb des Weltsystems. »Der Süden «, der noch in den 70er Jahren dazu in der Lage war, mit relativ einheitlicher Stimme für eine Neue Weltwirtschaftsordnung zu streiten, ist Mitte der 90er Jahre vollständig fragmentiert. Die UNCTAD, einst Sprachrohr und think tank der Entwicklungsländer, hat an Einfluß verloren. Der OECD ist also nicht nur der
»Feind im Osten «, sondern auch die handlungsfähige Interessenorganisationen des Südens abhanden gekommen. Dies ist selbst aus der Sicht der Industrieländer kein Vorteil, da es an Strukturen zur Kanalisierung und Lösung von Konflikten und Problemen im Nord-Süd-Verhältnis mangelt. Und letztlich haben sich die Machtgewichte auch innerhalb des Nordens beachtlich verschoben. Teile Asiens bilden heute das dynamischste Zentrum der Weltwirtschaft. Japan ist zu der Ökonomie geworden, die - wie die USA Anfang der 50er Jahre - in Schlüsselindustrien international die technologischen und organisatorischen Standards setzt. Die erste Generation der ostasiatischen Schwellenländer hat das wirtschaftliche Niveau der südeuropäischen Länder zum Teil schon überstiegen. Welche Stellung China in ein oder zwei Dekaden in der Weltwirtschaft einnehmen wird, ist schwer vorauszusagen. Die Auswirkungen dieser Dynamik auf die Weltordnung sind bisher völlig unklar. Der Westen die Alte Welt und Nordamerika hat sich lange für die Inkarnation »des Nordens« gehalten. Und die Länder Ostasiens beginnen erst seit kurzem, über ihre zukünftige Rolle im internationalen System nachzudenken.

Trotz dieser »ostasiatischen Newcomer《 ist die Welt eine »Vier-Fünftel-Welt«: $80 \%$ der Weltbevölkerung verfügen über $20 \%$, und $20 \%$ der Weltbevölkerung über $80 \%$ des 
global erzeugten Sozialprodukts. Und die Entwicklungs- und Wohlstandsunterschiede werden größer. Für die daraus entstehenden Konflikte gibt es bis heute keine Lösung, da die Ursachen der Ungleichheit tabu zu sein scheinen: die gesellschaftlichen Formen der Akkumulation nämlich. Sie haben sich ja gerade im Systemwettbewerb als überlegen herausgestellt, warum sollten sie geändert werden.

Man weiß nur: Die Methoden der Entwicklungspolitik, wie sie in den Jahrzehnten nach dem Zweiten Weltkrieg im Zuge der Modernisierungsstrategien angewendet wurden, sind gescheitert. Die Währungsbeziehungen, die von 1944 bis 1971 so erfolgreich stabilisiert worden waren, sind in der Mitte der 90er Jahre instabiler denn je. Dies hat natürlich damit zu tun, daß die Weltfinanzbeziehungen völlig unreguliert sind. Tagtäglich wechseln 1000 Milliarden US-Dollar auf den Devisenmärkten die Hände; gegen deren destabilisierende Wirkungen kann keine Zentralbank, und sei sie noch so mächtig, etwas ausrichten. Der Ökonomie-Nobelpreisträger von 1981, James Tobin, hat bereits 1978 vorgeschlagen internationale $\mathrm{Fi}^{-}$ nanztransaktionen weltweit zu besteuern, um Wechselkurs- und Zins-spekulationen einzudämmen. Er appelliert im Human Development Bericht des UN-Development Progamms 1994 dringend an die internationalen Finanzinstitutionen, mit Maßnahmen dieser Art der interna- tionalen Finanzspekulation, die erheblich zur Unterminierung nationaler Geldpolitik und nichts zu langfristig orientierten Investitionen beiträgt, Paroli zu bieten.

Doch dies ist Zukunftmusik. IWF und Weltbank haben in den vergangenen 5 Jahrzehnten nur ihren $\mathrm{Na}$ men behalten. Ihre Funktionen haben sich grundlegend gewandelt. Sie sichern heute die instabilen Finanzmärkte gegen selbstproduzierte Krisen, und sie sorgen dafür, daß die Öffnung aller Länder zum Weltmarkt durch strukturelle und sektorale Anpassungsmaßnahmen abgesichert wird. Das GATT hat seinen Auftrag, dem Prinzip des Freihandels auf dem Globus zur Durchsetzung zu verhelfen, in der jüngsten Uruguay-Runde beinahe 100prozentig erfüllt. Denn nun sind auch Dinge zum Gegenstand des Freihandelsprinzips gemacht worden, die bislang gar keine international handelbaren Waren sein konnten, weil die Eigentumsrechte an ihnen nicht geklärt waren. Aber nun sind Dienstleistungen und intellektuelle Eigentumsrechte dem Reglement des Freihandels unterworfen. Das GATT hat sozusagen seine Schuldigkeit getan und gibt den Staffelstab an die Welthandelsorganisation weiter. Diese jedoch wird sich mit tatsächlichen Neuerungen von Weltmarkt und Weltgesellschaft auseinandersetzen müssen: Erstens mit der zunehmenden $\gg$ Regionalisierung der Weltgesellschaft« (vgl. dazu PROKLA 90, März 1993) und der »Tri- 
adenkonkurrenz « und zweitens mit den Herausforderungen, die auf der UNCED-Konferenz 1992 in Rio de Janeiro formuliert worden sind. Wenn Wirtschaften generell »sustainable « sein soll, dann kann der Handel davon ebensowenig ausgeschlossen werden wie das Währungssystem oder der globale Finanzmarkt, es sei denn, man nimmt $\mathrm{zu}$ der gewagten Hypothese Zuflucht (wie sie von Neoklassikern so gern aufgestellt wird), daß Handel per se umweltschonend und daher weltweiter Freihandel der beste Umweltschutz sei.

III.

Eine »neue Weltordnung « existiert also keineswegs, aber es ist auch klar, daß die Strukturen und Organisationen der alten Weltordnung nicht mehr tragen. Die Welt befindet sich in einer Übergangsepoche. An welchen Bifurkationen welche Wege eingeschlagen werden, ist keineswegs ausgemacht und es kann erst recht nicht ausgeschlossen werden, daß es sich um Sackgassen handelt. Seltsam mutet es an, daß die herrschende Politik sich bei der Suche nach neuen Ordnungselementen und bei der Reorganisation internationaler Strukturen Begriffsmuster bedient, die vor einigen Jahren noch von sozialen Bewegungen und kritischen Wissenschaftlern gegen den »mainstream « in Wissenschaft und Politik hochgehalten wurden. Nun werden die Begriffe »entkernt $\ll$ (Beck) und mit einem nachgerade konträren Gehalt gefüllt. Nehmen wir den Begriff der »erweiterten Sicherheit «, der bewußt die vernachlässigten nicht-militärischen Elemente von Sicherheitspolitik in den Vordergrund rückte. Früher hätte man das als Entmilitarisierung von Sicherheitspolitik verstehen können und sollen. Heute allerdings zeigt es sich, wie unter dem Signum der »Entkernung « des Begriffs der Entmilitarisierung und Abrüstung eine Militarisierung von bislang nicht militärischen Handlungsfeldern von Politik herauskommt.

In den verteidigungspolitischen Richtlinien der Bundeswehr vom November 1992 wird von der Notwendigkeit der $\gg$ Sicherung der Rohstoffwege« gesprochen, von denen das reibungslose Funktionieren der Industriesysteme abhängig ist. Die Gesellschaften mit »OECD-Profil« greifen nicht nur ökonomisch auf die energetischen und materialen Rohstofflager $\mathrm{zu}$, sie bereiten sich auch auf die militärische Sicherung der $\gg$ just in time «-Logistik vor. Der Bundeswehrgeneral

Naumann sprach obendrein von der Bedrohung des Nordens durch ökologische Krisen im Süden, die beispielsweise Migrationsbewegungen auslösen könnten. Die Entwicklung der sicherheitspolitischen Strukturen ist jedenfalls so weit abgeschlossen, daß in ihr neue interventionistische Strategien erkennbar sind (vgl. den Beitrag von Helmut Hugler). Auf die UNO kommen in diesem Kontext ganz neue Funktionen zu (vgl. 
den Beitrag von Ulrich Albrecht). Inwieweit der selektive Interventionismus tatsächlich der Wiederherstellung von Menschenrechten und der Durchsetzung des Völkerrechts verpflichtet ist oder ob er nicht doch in erster Linie von »strategischen Interessen der OECD-Länder $a b$ hängt, ist seit dem zweiten Golfkrieg auch in der Linken umstritten. Daß es 1991 am Golf nicht einfach nur um die Sanktion einer Verletzung des internationalen Rechts und um die Wiederherstellung der nationalen Souveränität eines UNO-Mitglieds ging, das durch ein anderes UNO-Mitglied annektiert worden war, sondern auch um öl, ist von Realpolitikern wie Richard Nixon oder Henry Kissinger in aller Offenheit gesagt und von kritischen Analytikern des Golfkonflikts auch gegen einen linken »mainstream《 belegt worden (vgl. dazu Beiträge in PROKLA 82 und PROKLA 84 vom März und September 1991). In den Beiträgen von Mariano Aguirre und Rüdiger Göbel wird diese Debatte über die Bedeutung der Interventionspolitik und die Rolle der UNO fortgesetzt.

Mit dem Ende des Ost-West-Konflikts ist zwar die Gefahr der von E. P. Thompson so genannten »exterministischen« Menschheitsbedrohung geschwunden und SDI erscheint in den 90er Jahren wie eine monströse Ausgeburt verrückter Phantasien. Inzwischen sind aber eine Reihe von neuen Konflikten entfesselt worden, die die Vorstellung von einer heraufziehenden Friedenszeit sehr schnell widerlegten.

Da sind die ethno-nationalistischen Konflikte, die zur Vorstellung von "ziviler i internationaler Politik nicht mehr passen. Das scheinbar so zivilisierte Europa, das zuweilen voller Überheblichkeit auf andere Weltregionen blickte, ist stellenweise auf längst überwunden geglaubte Formen der Konfliktaustragung zurückgefallen. Kann man ethno-nationalistische Konflikte mit den politischen Methoden der alten oder der neuen Weltordnung lösen? Sind die sicherheitsstrategischen und militärischen Konzepte geeignet, um die Kriege im Kaukasus und auf dem Balkan zu beenden, den Ausbruch von Konflikten im Baltikum und anderswo in der ehemaligen Sowjetunion $\mathrm{zu}$ verhindern? Es sind Zweifel angebracht. Das Macho-Gehabe von NATO und UNO-Entscheidungsträgern erscheint ebenso unangemessen wie die primitiven Versuche in der Tagespresse (als Beispiel nehme man nur die »taz«) die Konflikte umzudefinieren: Im Bürgerkrieg auf dem Balkan wird schlicht eine Partei zum Aggressor deklariert, um die Auseinandersetzung mit den innergesellschaftlichen Konfliktursachen zu ersparen und wieder in die einfache Welt nationalstaatlicher Grenzziehungen zurückkehren zu können. Wie beruhigend ist es doch, Staaten an einem Territorium festzumachen, und wenn Kriege auf einem Territorium geführt werden, diesen Krieg 
als Aggression eines anderen Territorialstaats definieren zu können. Das ist der Versuch, mit der Vorstellungswelt des 19. Jahrunderts die Konflikte an der Schwelle zum 21. Jahrhundert zu analysieren.

Da sind aber auch die sich bereits heute abzeichnenden Konflikte um die begrenzte Natur auf Erden. Hier schürzen sich alle Bedingungen für einen neuen Nord-Süd-Konflikt um die Nutzung von Ressourcen und die Belastung von Senken (vgl. dazu den Beitrag von Elmar Altvater). Es geht um einen Kampf um positionelle Güter, über die eben nicht alle Welt, sondern nur ein Teil der Welt verfügen kann. Rationierung von Umweltgütern wird zu einem Prinzip, bei dem lediglich offen ist, ob es durch den Marktmechanismus oder durch politische Macht oder gar militärisch zur Geltung gebracht wird. Die Gefahr besteht, daß sich die $\gg$ Vier-Fünftel-Welt « zu einem Apartheid-Globus verfestigt. Von einem Konsens über die Nutzung von Umweltgütern sind wir trotz UNCED-Konferenz in Rio de Janeiro weit entfernt.

Die Tendenzen zur Herausbildung einer internationalen »Zivilgesellschaft « sind jedenfalls schwach. Die international operierenden Institutionen und Organisationen haben einen nicht geringen Nachholbedarf an demokratischer Legitimierung. Nicht weil dies von Demokratietheoretikern so verordnet worden wäre, sondern weil sich dieses Demokratiedefizit bei der Umsetzung von Beschlüssen, bei der Verfolgung von Perspektiven, bei der Durchsetzung von Entscheidungen als Mangel erweist.

DaB sich Interaktionen und Strukturen im historischen Kapitalismus auf der globalen Ebene verdichten, ist offensichtlich. Von einer tatsächlichen »Weltgesellschaft « zu sprechen, wäre aber sicherlich verfrüht. Dem sich vollziehenden Wandel muß selbstverständlich auch die Theorie Rechnung tragen, also auch die Wissenschaft von den internationalen Beziehungen, deren Begriffe und Konzepte zu überprüfen sind (vgl. den Beitrag von Christoph Scherrer). Die geforderte Erneuerung nach dem Ende der alten Weltordnung ist also vieldimensional: In der Sicherheitspolitik wie bei den internationalen Institutionen, im Umgang mit den ökologischen Problemen und bei der Gestaltung einer Weltwirtschaftsordnung, bei den gewohnten Begriffen, die dekonstruiert und neu zusammengesetzt werden müssen, um die Dynamik der heraufdämmernden »neuen Weltordnung « zu begreifen. 